differentiation with gouty or rheumatic affections. In the earlier stages the disease may be and of ten is confounded with these two last named inflammations ; an examination of the fluids and the progressive character of arthritis, if not arrested, will make diagnosis easy.

Where the manifestation is in the alveolar-dental articulation it probably has its origin in the trophic center in the spinal cord corresponding to the part affected, and I see no reason why this inflammation may not make its first appearance in the dental articulation, as in any other articular surface. To the pocket once formed there is added those septic complications, varying with the habits of the individuals affected, that produce a likeness and make the difficulty of diagnosis greater.

When a better knowledge of the nervous relations of the teeth has been acquired, I feel quite certain that many doubtful oral conditions will be better understood and properly classified. In this connection I am impressed with the necessity for instruction in neurology as a part of the curriculum of our modern dental schools, where scientific training is paramount to purely constructive work.

In reference to gouty manifestations in the oral cavity, the recently published results of the inves. tigations of Drs. Pierce, Kirk, Burchard, Faught and others furnish such a fund of information upon the subject as to leave no doubt in regard to the effects of retained uric acid in the circulating fluids.

In the light of the before mentioned theory of Moleschott, we can understand how the peridental membrane may become the seat of such deposits as urates of lime and soda. The office of the membranous groups represented by the periosteum and pericementum is the deposition of lime salts. In mild inflammatory conditions, not accompanied by perversion of the circulating fluids, there is developed an activity in the pericementum that results in an increased deposition of cement beyond the typal limits of the root in similar inflammations of this membrane, caused by or accompanied with an excess of urates in solution in the circulating fluids. We can understand how a deposition of salts of calcium may be made that would show upon analysis the presence of urates; indeed, we have no assurance that the presence of morbific materials in the blood would not cause the specific inflammation that induces the deposit with its inflammatory coloring.

In my experience, the deposit known as serumal calculus is not removable by any means at our command that would not be equally destructive to the surrounding tissues. In pockets of long standing freely accessible to the fluids of the mouth and to oral débris, there may be an added deposit on the surface of a root in granular form that is removable. This, however, is only manifested in the last stages of the disease when re-attachment is doubtful.

Where the pocket is manifested on but one side, and all removable deposit has been carefully taken away, I do not think that the loss of that limited area of peridental membrane offers any barrier to return to health of the surrounding tissue and entire attachment of the tooth.

It was my intention to have presented something more upon the gouty diathesis, but as the ground has been so well covered in recent publications in our professional journals, I shall not hope to bring you anything new, and trust the discussion may more fully develop a subject fraught with so much of interest.

How much the uric acid diathesis has to do with other destructive oral manifestations has not been determined, but I am convinced that some specific acid condition of the circulating fluids is the cause of that mysterious wasting away of the enamel so frequently met with, and which in description of what really occurs, we denominate erosion. What we now recognize as alveolar pyorrhea in its several manifestations, I have shown, or endeavored to show to be due to any one of two or three systemic conditions requiring a different classification and a nomenclature suggesting in part its origin. When this has come to be scientifically developed, an entirely new system of treatment for each will be evolved. Already the study of this subject by dental practitioners has been the means of introducing an entirely new salt of lithium, as an addition to the materia medica. I refer to the preparation known as tartar]ithin or lithium bi-tartrate, first made by Dr. E. C. Kirk, of Philadelphia, a practicing dentist and an expert medical chemist. In fact all the information we have up to this time upon the oral manifestations of the uric acid diathesis is due to dental investigation. What the dentist can do for these conditions will depend upon his preparation for practice and his conception of its scope; certainly instrumentation is not to be entirely relied upon. Primarily this was designed to be only a process of cleansing and not instrumentation in its surgical sense. To be entirely euccessful therefore means a wider knowledge of systematic conditions and also the ability to recognize the pathologic significance of oral lesion. Are dentists properly prepared for such work? Or does the purely constructive or prosthetic secure the largest share of attention in our technical schools? We think that the curriculi of our modern schools of the best class comprise all that is essential for such a training. Where they do not, the instruction in physiology, pathology, and histology can be strengthened; if not, the schools of medicine are open for post-graduate instruction with liberal allowances for work already done, thus enabling the dental graduate to complete his preparation for practice upon its highest plane.

DOUBLE NASAL ATRESIA DUE TO SMALLPOX. Read before the Mississippi Valley Medical Assoc'ation, at Hot Springs, Nov. 20, 1894

BY HANAU W. LOEB, A.M., M.D.

$$
\text { ST. Lours, мo. }
$$

One-sided nasal atresia due to smallpox is uncommon enough in rhinologic literature, and as double nasal atresia caused by smallpox must be all the rarer, I am prompted to present the details of this most interesting case to the Association. It is all the more desirable for presentation on account of the success of the treatment, and the great relief which the patient has experienced, for we. all know the difficulty of maintaining an opening which nature once has closed.

We read, with remarkable monotony, of failure after failure in the treatment of webbed fingers and conditions due to the deposit of scar tissue; we realize quite readily the almost futility of the treatment for the permanent relief of cicatricial contractions and adhesions, resulting from syphilitic lesions of 
the pharynx, palate and larynx, so that while no great skill is claimed in the management of the case in point, yet the patient's condition after a year and a half of observation certainly merits attention:

Miss E. T., age 30 years, consulted me first on April 6, 1893, with the following history: Twenty-nine years ago she was subjected to a severe attack of smallpox, which very seriously affected her health. The disease was of the confluent type, and exhibited itself more upon her face than any other portion of her body. There had evidently been a coalescence of a number of pustules over the alae of the nose, and the portion of the lip which is in immediate relation with the nose. Considerable scarring naturally resulted, mainly exhibited on the face, especially in the region of the nose and lips. She had several scars upon her body, arms and lower extremities, but none manifest the degree of cicatrization that is to be found in those upon the face. The result of this loss of tissue was a very decided contraction of the nasal orifices, so that they were no larger than a broom straw.

Five years afterward, an attempt was made by a prominent surgeon to enlarge the openings of the nostrils. For some reason the only effect was to completely obliterate the openings; so that for the past twenty years she has been absolutely unable to breathe through her nose.

Since that time the patient has been in fairly good health,

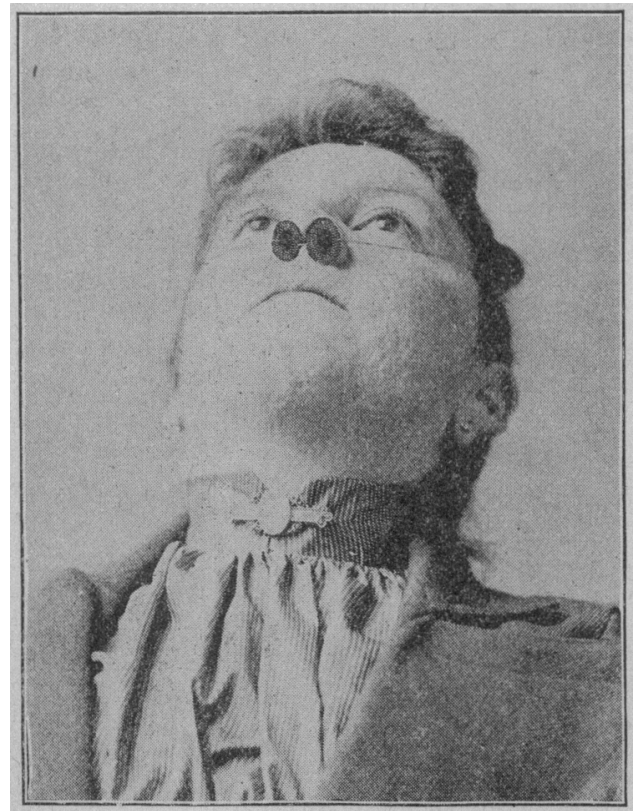

with the exception that she has been suffering from a dry throat which was especially severe early in the morning; so severe in fact, that she was prevented from partaking of an early morning meal. She suffered very frequently from headaches, which were localized to no particular part of the head. Strangely enough she was not subject to acute pharyngitis though ber throat, she said, was a source of continual trouble. She has not been subject to bronchitis, and she has never had pneumonia or any serious illness since her attack of small-pox. During the two years previous to the time she consulted me, her sym ptoms were somewhat aggravated, and the dryness about her throat had increased. The hawking and clearing of her throat which were almost continually a source of annoyance to her were decidedly increased, and, as she expressed it, she was much sicker during this time than before. She fails to relish her food from a lack of taste.

Examination revealed very plainly the effect of the attack of smallpox. Numerous scars were to be found like the ordinary marks resulting from the disease but, as has been stated before, they were larger and more prominent on the face. Examination of the pharynx showed a chronic inflamed pharynx of a mild type, and with numerous spots of folliculous inflammation. The naso-pharynx was also affected with a chronic inflammation, but the larynx did not exhibit any evidence of disease. It was made very man- ifest that behind the point of atresia the nasal cavities were open and free,for the patient could easily balloon out the soft parts by forcing air from the pharynx into the nose. In this way too, it could be shown that the overgrowing tissue was perfectly impervious, for the swelling produced by blowing was not reduced until the patient ceased her efforts. The voice was quite clear, and indeed it was only by close attention that an obstruction would be expected.

The patient, having consented to an operation, was sent to the Rebekah Hospital, and upon April 27, Dr. H. C. Dalton operated in the following manner: After aseptic preliminaries, an incision about half an inch long was made through the cicatricial tissue, somewhat larger on the left side than upon the right. It was necessary to make a larger incision upon the left side, from the fact that the left ala had collapsed considerably on account of a greater destruction of the alar cartilage upon that side. The skin and mucous membrane were then stitched together, around the improvised openings, leaving on each side a fairly normal looking orifice. Into each nostril was introduced a rubber tube, devised by myself after the plan of Simrock's speculum, being, however, about one third larger, and having a larger rim or flange. Into this flange four small openings were made for the purpose of inserting threads. The two tubes were sutured to the adjacent skin, so as to be certain that they would be held in place. The whole was covered with

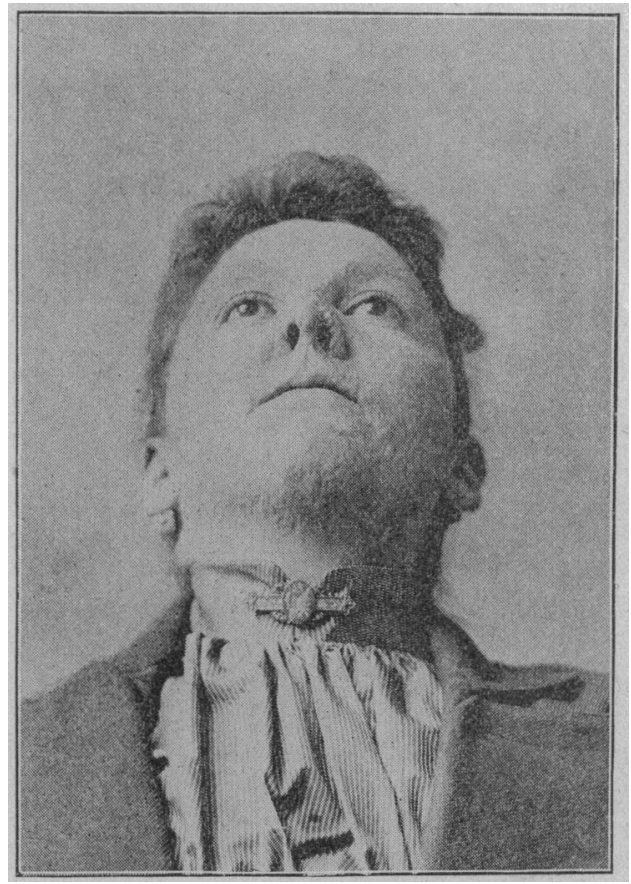

gauze, and the patient put to bed. She suffered from very slight inconvenience, and after four days the sutures holding the tubes in position were removed, and the whole nose subjected to a thorough cleansing, after which the tubes were re-introduced.

They were now beld in position in a somewhat different manner. The thread was placed in the two inner openings, bolding them close together, and another thread into each of the two outer openings in the flange and these were carried behind the head and held firmly by a hairpin. This method was found to be eminently satisfactory, very slight displacement being possitble. The tubes were easily kept in place, and from their form were not at all inconvenient or uncomfortable to the patient. She left the hospital after three weeks, the wound being entirely healed, and the margin of the new nostrils being smooth and normal. She continued to wear these tubes constantly for three months, being warned of the danger of a reunion of the two sides of the nostrils. In three months she was permitted to leave the tubes out for an hour at a time; later this time was extended. Meanwhile the patient was looking very much better, and as a matter of course, she suffered from no dryness of the throat, and from none of the other symptoms which were so unpleasant to her. Her headaches entirely disappeared, and her sense of smell returned. She was able to 
appreciate the odor of flowers three days after the operation. On March 31, 1894, a second operation was performed upon the left side, as it was evidently not so satisiactory as the right nostril, a fact easily explained when it is remembered that so much of the alar cartilage was destroyed. The incision was carried somewhat higher up toward the tip of the nostril than it had been, and a slightly larger tube was as inserted. This operation has had a decidedly good effect, and now the left nostril is almost as perfect as the right. Since July 1, she has not worn the tubes during the day, now four months. Examination on September 15 revealed no evidence of any return whatever to the original condition. It was suggested to her that she might leave the tubes out altogether, but since she is rather fearful lest her old condition might return, she prefers, at least for the time, to wear them every night.

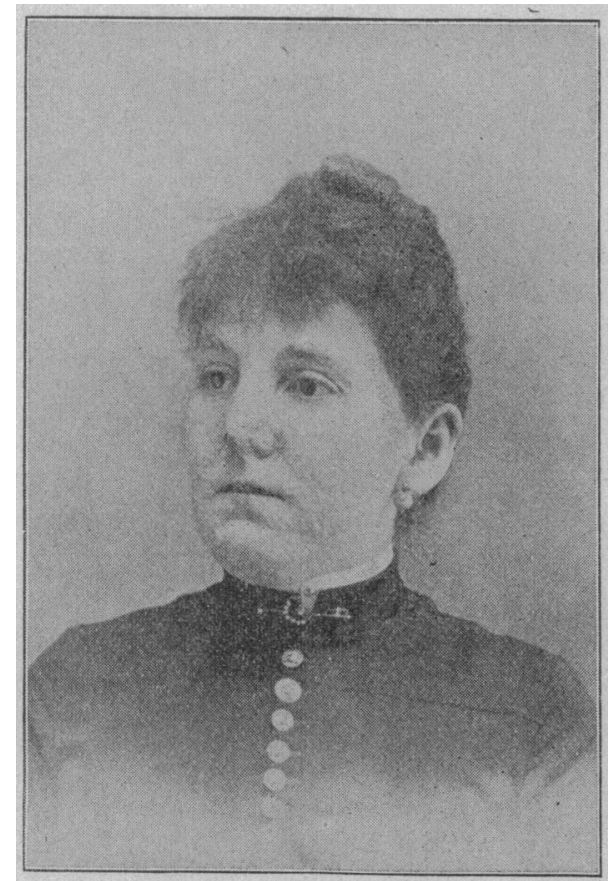

The illustrations which are presented, exhibit the appearance of the case with the tubes inserted, and the appearance of the nostril a year and a half after treatment. The picture showing the condition of the nose before the operation is not a very satisfactory one, being taken from an old photograph, of some ten years ago.

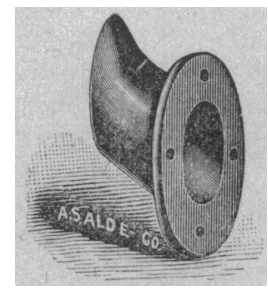

I am quite certain that the good result in the present case has been due to two circumstances: In the first place the mucous membrane and the skin were united, the skin being rather redundant, and therefore capable of forming a good protection for the margin of the nostrils. If there had been less skin, I am quite certain that the result would not have been quite so pleasing, but with the redundant tissue it was reasonable to expect so good a result. In the second place, the tubes which she wore subjected the nose to no pressure, and maintained their position without any degree of irritation. They fulfilled their function so nicely, that I would commend a similar tube in any case of a like nature.

321 North Grand Avenue.

\section{CASE OF FRACTURE OF ANATOMIC NECK} OF THE HUMERUS.

BY E. L. GIBSON, M.D. STAUNTON, VA.

A 16-year-old boy, of good build, fell about twenty feet into some ladders. I saw him about two hours after the injury; he complained of great pain at the shoulder joint. A slight contusion was seen on the front of the arm at the shoulder joint; I was unable to find any other contusion.

On superficial examination, I thought I had a sub. spinous dislocation; but the protrusion did not look as large as would be expected. To isolate fracture, I placed the fingers of one hand on the bicipital tuberosities and rotated the humerus; they were felt to move under my fingers; no crepitation was elicited in this way. Dr. J. St. P. Gibson saw him at this time and confirmed my diagnosis. He was given chloroform; reduction was effected by Koker's method after several trials. Rotation was now made and erepitation was very perceptible.

Treatment: Gibson's wire splint, which was described in the Trausactions of the Ninth International Medical Congress,Vol. I., page 585, was applied.

By this means, extension was continued for four weeks, at which time the wire splint was removed and a pasteboard crown splint was applied and allowed to remain two weeks. Upon its removal the deltoid muscle was found much atrophied; allowing the head of the humerus to be examined thoroughly. A line of callus could be felt. The joint admitted of all motions without pain and a perfect shoulder is the result. The crown splint will be retained for a couple of weeks longer, allowing slight motion.

\section{SANITARY LEGISLATION.}

Abstract of Remarks before the Auxiliary Health Association, Spring. field, Ill.

BY CLARKE GAPEN, M.D., LL.B.

KANKAKEE, ILL.

As I look back over the twenty years of my life and work in medicine, I find that nothing has grown upon me more steadily or forcibly than the belief that the future of medicine is to be largely in the field of sanitation. The medical man who bas kept himself abreast with the times has seen one line of treatment and one theory after another give way. My worthy preceptor who presides here, twenty years ago taught me many things that to-day he would declare worthless. All of our teachers have taught us much with regard to medical treatment which has since vanished into thin air like the baseless fabric of a vision, but in all this time sanitation has steadily grown in importance until to-day in practical significance it is not secondary to any other thing, even in medical instruction.

The fact that my experience in the line of sanitary work has been somewhat extended and peculiar, is my only excuse for offering ideas of so positive a character as those which I now present. Beginning with_an almost absolute sanitary chaos, in a city of 